\title{
Pretreatment with a combination of ligustrazine and berberine improves cardiac function in rats with coronary microembolization
}

\author{
Ying ZHANG ${ }^{1,2,3}$, Xiao-juan MA ${ }^{2,3, *}$, Chun-yu GUO ${ }^{4}$, Ming-ming WANG ${ }^{2,3}$, Na KOU ${ }^{1,2,3}$, Hua QU ${ }^{1,2,3}$, Hui-min MAO ${ }^{1,2,3}$,
} Da-zhuo $\mathrm{SHI}^{2,3, *}$

${ }^{1}$ Graduate School, Beijing University of Chinese Medicine, Beijing 100029, China; ${ }^{2}$ China Heart Institute of Chinese Medicine, China Academy of Chinese Medical Sciences, Beijing 100700, China; ${ }^{3}$ Cardiovascular Diseases Center, Xiyuan Hospital, China Academy of Chinese Medical Sciences, Beijing 100091, China; ${ }^{4}$ Beijing Obstetrics and Gynecology Hospital, Capital Medical University, Beijing 100020, China

Aim: We have shown that a combination of ligustrazine and berberine produces more effective inhibition on platelet activation and inflammatory reactions in rat acute myocardial infarction compared with either agent alone. In this study we evaluated the beneficial effects of a combination of ligustrazine and berberine in a rat model of coronary microembolization (CME).

Methods: SD rats were treated with ligustrazine, berberine, ligustrazine+berberine, or clopidogrel for 2 weeks. When the treatment completed, CME was induced by injection of sodium laurate into the left ventricular, while obstructing the ascending aorta. All rats were intubated for hemodynamic measurements. Blood samples were collected for biochemical analyses, flow cytometry, and ELISAs. Heart tissues were isolated for histopathology and subsequent protein analyses.

Results: Pretreatment with the combination of ligustrazine $\left(27 \mathrm{mg} \cdot \mathrm{kg}^{-1} \cdot \mathrm{d}^{-1}\right)$ and berberine $\left(90 \mathrm{mg} \cdot \mathrm{kg}^{-1} \cdot \mathrm{d}^{-1}\right)$ significantly improved cardiac function, and decreased myocardial necrosis, inflammatory cell infiltration, microthrombosis and serum CK-MB levels in CME rats. In addition, this combination significantly decreased plasma ET-1 levels and von Willebrand factor, inhibited ADP-induced platelet activation, and reduced TNF $\alpha$, IL-1 $\beta$, ICAM-1 and RANTES levels in serum and heart tissues. The protective effects of this combination were more prominent than those of ligustrazine or berberine alone, but comparable to those of a positive control clopidogrel (6.75 $\left.\mathrm{mg} \cdot \mathrm{kg}^{-1} \cdot \mathrm{d}^{-1}\right)$.

Conclusion: The combination of ligustrazine and berberine significantly improved cardiac function in rat CME model via a mechanism involving antiplatelet and anti-inflammatory effects.

Keywords: coronary microembolization; ligustrazine; berberine; clopidogrel; cardioprotection; platelet activation; inflammatory reactions

Acta Pharmacologica Sinica (2016) 37: 463-472; doi: 10.1038/aps.2015.147; published online 29 Feb 2016

\section{Introduction}

Coronary microembolization (CME) is a potential cause of cardiac dysfunction in the absence of atherosclerotic obstruction of the epicardial coronary artery. Decades ago, CME was first identified at autopsy of patients dying from a sudden cardiac event $^{[1]}$. However, CME is now more frequently observed following thrombolytic therapy and coronary interventions for an in situ coronary thrombus, or the spontaneous lysis of a remote arteriole thrombus; these may result in myocardial

\footnotetext{
* To whom correspondence should be addressed.

E-mail shidazhuo@126.com (Da-zhuo SHI); abc_mxj@aliyun.com (Xiao-juan MA)

Received 2015-09-21 Accepted 2015-12-15
}

micronecrosis, cardiac dysfunction, arrhythmias, and coronary reserve reduction ${ }^{[2]}$. CME is a main cause of non-obstructive coronary artery disease. One study ${ }^{[3]}$ showed that patients with non-obstructive coronary artery disease were also at an elevated risk for cardiovascular events. An increasing number of studies ${ }^{[4-5]}$ have shown that platelet activation is critical in the formation of in situ coronary thrombi and that the subsequent inflammatory reaction is a main contributor to contractile dysfunction.

Ligustrazine is extracted from the roots and stems of Ligusticum chuanxiong Hort (Chuan Xiong). Previous studies demonstrated that ligustrazine inhibited platelet activation induced by adenosine diphosphate $(\mathrm{ADP})^{[6-7]}$. However, these studies did not explore this inhibitory effect in vivo. In vivo research 
suggested that ligustrazine improved cardiac function in rats with heart failure by inhibiting myocardial cell apoptosis and cardiomyocyte hypertrophy ${ }^{[8-9]}$. Berberine is an important alkaloid derived from Coptis chinensis Franch (Huang Lian). Previous studies ${ }^{[10-11]}$ indicated that berberine had cardioprotective effects that included lowering lipids, reducing ischemia/reperfusion-induced myocardial apoptosis, and attenuating adverse left ventricular remodeling ${ }^{[12-14]}$. Our previous study in rats with acute myocardial infarction ${ }^{[15]}$ showed that combined ligustrazine and berberine produced more effective inhibition of platelet activation and inflammatory reactions than did either agent separately. However, the effects of ligustrazine plus berberine have not been examined in rats with CME.

In the present study, we established a rat model of CME and hypothesized that treatment with ligustrazine plus berberine would improve cardiac function by inhibiting platelet activation and the consequent inflammatory cascade.

\section{Materials and methods}

\section{Drugs and reagents}

Ligustrazine was provided by the Beijing Yan Jing Pharmaceutical Co Ltd (Beijing, China). Berberine was purchased from the Beijing Cheng Ji Pharmaceutical Co Ltd (Beijing, China). Clopidogrel sulfate tablets were purchased from SanofiAventis (Hangzhou, China). Sodium laurate was obtained from Sigma-Aldrich (St Louis, MO, USA). Enzyme-linked immunosorbent assay (ELISA) kits for endothelin-1 (ET-1), von Willebrand factor (vWF), P-selectin, cyclic AMP (cAMP), tumor necrosis factor alpha (TNFa), interleukin-1 beta (IL-1 $\beta$ ), intracellular adhesion molecule-1 (ICAM-1), and regulated on activation, normal $\mathrm{T}$ cell-expressed and secreted (RANTES) were obtained from R\&D Systems (Minneapolis, MN, USA). Vasodilator-stimulated phosphoprotein phosphorylation (VASP-P) kits (PLT VASP/P2Y ${ }_{12}$ ) were purchased from Biocytex (Marseille, France). Antibodies against TNFa, IL-1 $\beta$, ICAM-1, RANTES, and glyceraldehyde 3-phosphate dehydrogenase (GAPDH) were purchased from Abcam (Cambridge, MA, USA).

\section{Animals}

Adult male Sprague-Dawley rats (approximately 300-350 g) were provided by the Academy of Military Medical Sciences Laboratory Animal Center (certificate number SCXK-(Jun) 2012-0004). Animals were acclimated for at least one week in standard conditions and given free access to a standard diet and distilled water. All procedures were approved by the Animal Care and Use Committee of Xiyuan Hospital, China Academy of Chinese Medical Sciences, and conformed to the Animal Management Rule of the Ministry of Health, People's Republic of China (Document 55, 2001).

\section{CME rat model and treatments}

Experimental CME can be induced by injecting homologous microthrombotic particles based on embolization from a distant source following spontaneous $\operatorname{lysis}^{[16-17]}$. The present study established a CME model by left ventricle (LV) injection of sodium laurate, as previously described ${ }^{[18-19]}$. This model was characterized by the formation of in situ coronary thrombi. Ninety male rats were allocated randomly to six groups: sham, CME model, ligustrazine $\left(27 \mathrm{mg} \cdot \mathrm{kg}^{-1} \cdot \mathrm{d}^{-1}\right)$, berberine $(90$ $\left.\mathrm{mg} \cdot \mathrm{kg}^{-1} \cdot \mathrm{d}^{-1}\right)$, ligustrazine + berberine $\left(\mathrm{L}+\mathrm{B} ; 27 \mathrm{mg} \cdot \mathrm{kg}^{-1} \cdot \mathrm{d}^{-1}+90\right.$ $\mathrm{mg} \cdot \mathrm{kg}^{-1} \cdot \mathrm{d}^{-1}$, respectively), and clopidogrel (positive control; $\left.6.75 \mathrm{mg} \cdot \mathrm{kg}^{-1} \cdot \mathrm{d}^{-1}\right)$. The indicated drugs were administered daily by oral gavage for two weeks, with the sham and CME model groups receiving vehicle. Twelve hours after the last treatment, the animals were anesthetized with pentobarbital sodium $(40 \mathrm{mg} / \mathrm{kg}$, intraperitoneally) and ventilated with a small animal ventilator (Chengdu Taimeng Software Co Ltd, Chengdu, China). A thoracotomy was performed at the midline of the chest, followed by a sternotomy between the second and third intercostal space. The pericardium was opened and the ascending aorta exposed fully. Sodium laurate $(2 \mathrm{~g} / \mathrm{L}$, $0.2 \mathrm{~mL}$ ) was injected as a bolus into the LV using a 29-gauge needle during a $30 \mathrm{~s}$ occlusion of the ascending aorta. For the sham group, an identical procedure was performed and $0.2 \mathrm{~mL}$ physiological saline was injected, instead of sodium laurate. The thoracic cavity and the skin incision were then closed with sutures. All rats were intubated $24 \mathrm{~h}$ after the operation to carry out hemodynamic measurements. Blood samples were collected for biochemical analyses, flow cytometry, and ELISAs. Hearts from five rats in each group were isolated for histopathology. The remaining hearts were frozen quickly for subsequent protein analyses.

\section{Hemodynamics}

Animals were anesthetized with pentobarbital sodium and arterial pressure was recorded by a polyethylene catheter (PE50), positioned in the right carotid artery. The catheter was then inserted into the LV to record the ventricular pressure. These data were collected using a pressure transducer coupled to a pressure amplifier. Pressure analog signals were digitized by a data acquisition and analysis system, with a sampling rate of $2000 \mathrm{~Hz}$. All equipment was from Chengdu Taimeng Software Company. These data were used to determine heart rate (HR), LV systolic pressure (LVSP), LV end-diastolic pressure (LVEDP), and LV maximum positive and negative pressure changes over time $\left(+\mathrm{d} p / \mathrm{d} t_{\max }\right.$ and $-\mathrm{d} p / \mathrm{d} t_{\max }$ respectively).

\section{Histopathological changes}

Hearts were harvested, fixed in $4 \%$ paraformaldehyde, embedded, and cut into $4-\mu \mathrm{m}$ sections from the injection point downwards, in parallel with the coronary ditch. Sections were stained with hematoxylin and eosin for the evaluation of inflammatory cell infiltration. Micronecrotic foci were identified with Heidenhain staining. The sections were scored for myocardial necrosis and cellular infiltration by a skilled and blinded observer. The scores were: 0 , no myocardial lesions; $1+$, lesions involving $<25 \%$ of the myocardium; $2+$, lesions involving $25 \%-50 \%$ of the myocardium; $3+$, lesions involving $50 \%-75 \%$ of the myocardium; $4+$, lesions involving $>75 \%$ of the myocardium. Microthrombosis was evaluated in sections 
using Carstairs staining, as described previously ${ }^{[18]}$. The number of microthrombi/100 microarteries (diameter $\leq 100 \mu \mathrm{m}$ ) was calculated for each group.

\section{Creatine kinase MB fraction (CK-MB)}

Blood was obtained from the abdominal aorta of all rats prior to sacrifice, centrifuged at $3500 \times g$ for $10 \mathrm{~min}$, and stored at $-80^{\circ} \mathrm{C}$. The serum levels of $\mathrm{CK}-\mathrm{MB}$ were determined using an automatic biochemical analyzer (Cobas8000, Roche).

\section{Flow cytometry analysis of VASP-P}

To measure ADP-induced platelet activation, the VASP239 phosphorylation state was determined in citrate-anticoagulated blood samples using the PLT VASP/P2Y $\mathrm{Y}_{12}$ kit, as described previously ${ }^{[20]}$. Samples were treated in accordance with the manufacturer's protocol and were analyzed within $4 \mathrm{~h}$ of collection. In brief, whole blood was incubated for 10 min at room temperature with either the solutions of $\mathrm{PGE}_{1}$ or $\mathrm{PGE}_{1}$ plus $10 \mu \mathrm{mol} / \mathrm{L}$ ADP that were provided with the kit. These blood samples were then treated with fixative containing paraformaldehyde. Platelets were permeabilized and incubated with either an anti-VASP-P mouse monoclonal antibody (16C2) or the negative isotype control provided with the kit. Samples were then incubated with a polyclonal antimouse IgG antibody conjugated to fluorescein isothiocyanate and a platelet counterstaining reagent-PE prior to immediate analysis by flow cytometry using a FACS Calibur (Becton Dickinson, San Jose, CA, USA). The platelet population was identified using the forward and side scatter distributions, and 5000 platelets were gated and collected. Data from the negative isotype control was used to correct the mean fluorescent intensities (MFIcs) of PGE $_{1}\left(\right.$ MFIC $_{\mathrm{PGE}}$ ) and $\mathrm{PGE}_{1}$ plus ADP $\left(\right.$ MFI $\left._{\mathrm{PGE}+\mathrm{ADP}}\right)$ samples that were incubated with anti-VASP-P. The platelet reactivity index (PRI) was calculated as outlined in the kit using the mean corrected fluorescence intensities and the following formula: PRI=[(MFI $\left.\left.\mathrm{C}_{\mathrm{PGE}}-\mathrm{MFI}_{\mathrm{PGE}+\mathrm{ADP}}\right) / \mathrm{MFI}_{\mathrm{C}_{\mathrm{PGE}}}\right]$ $\times 100$.

\section{ELISA determinations of ET-1 and vWF}

Plasma ET-1 and vWF levels were measured using an antigenbased sandwich ELISA, according to the manufacturer's instructions. For ET-1 measurements, 96-well plates were coated with $100 \mu \mathrm{L}$ of an anti-ET-1 monoclonal antibody per well. Between subsequent steps in the assay, the coated plates were washed three times in washing buffer. After a 30-min preincubation with washing buffer at $37^{\circ} \mathrm{C}, 100$ $\mu \mathrm{L}$ of standard ET-1, control solution, or rat plasma were added and incubated for $2 \mathrm{~h}$ at $37^{\circ} \mathrm{C}$. A goat polyclonal anti-murine ET-1 antibody was then added for $90 \mathrm{~min}$, followed by a horseradish peroxidase (HRP)-conjugated donkey anti-goat antibody for $1 \mathrm{~h}$, at room temperature. Diluted $o$-phenylenediamine $(100 \mu \mathrm{L})$ was then added. The enzymatic reaction was stopped by adding $100 \mu \mathrm{L} 1 \mathrm{~mol} / \mathrm{L}$ sulfuric acid, and the absorbance was then measured at $492 \mathrm{~nm}$ using a microplate spectrophotometer.

\section{P-selectin and cAMP determinations}

Plasma P-selectin and cAMP levels were determined using an antigen-based sandwich ELISA, according to the manufacturer's instructions.

\section{Inflammatory mediator levels}

Serum levels of TNFa, IL-1 $\beta$, ICAM-1, and RANTES were determined using an antigen-based sandwich ELISA, according to the manufacturer's instructions.

\section{Western blotting}

Frozen heart tissues were homogenized in cold RIPA buffer (50 mmol/L Tris, $150 \mathrm{mmol} / \mathrm{L} \mathrm{NaCl}, 2 \mathrm{mmol} / \mathrm{L}$ EDTA, 1\% Triton, $0.5 \%$ deoxycholic acid, $0.1 \%$ SDS, plus protease inhibitor cocktail) using a glass homogenizer. The homogenized tissues were centrifuged at $14000 \times \mathrm{g}$ for $30 \mathrm{~min}$ at $4^{\circ} \mathrm{C}$ and the protein concentrations of the supernatants were determined by Bradford assay. Samples were then mixed with $4 \times$ SDS buffer to a final dilution of $1 \times$, and heated to $95^{\circ} \mathrm{C}$ for $5 \mathrm{~min}$. Proteins were separated by electrophoresis using SDS-polyacrylamide gels and blotted onto $\mathrm{P}^{\mathrm{sq}}$ (RANTES, TNFa, and IL-1 $\beta$ ) or polyvinylidine fluoride (ICAM-1) membranes. Proteins were detected using rabbit polyclonal anti-RANTES (Abcam, ab9783), rabbit polyclonal anti-IL-1 $\beta$ (Abcam, ab9722), rabbit polyclonal anti-TNFa (Abcam, ab9755), or mouse monoclonal anti-ICAM-1 (Abcam, ab2213) antibodies. HRP-goat antimouse IgG (Jackson, 115-035-003) or HRP-goat anti-rabbit IgG (Pregene \#GR-100) were used as secondary antibodies, as appropriate. ImageJ software was used to analyze the intensity of the protein bands.

\section{Statistical analysis}

Data are expressed as mean \pm standard error of the mean (SEM). Significant differences were determined using oneway analysis of variance (ANOVA) followed by the Tukey post hoc test. A $P$ value less than 0.05 was considered significant. Statistical analyses were performed by SPSS 20.0 software (IBM, Chicago, IL, USA).

\section{Results}

\section{Histopathological findings}

The histopathological findings are listed in Table 1. In contrast to the sham group, the CME model group showed significant myocardial necrosis, with inflammatory cell infiltration and in situ coronary microthrombi $(P<0.01$, Figures $1-3)$. Myocardial necrosis, inflammatory cell infiltration, and in situ coronary microthrombi were decreased in the $\mathrm{L}+\mathrm{B}$ group, as compared with the CME model group $(P<0.01)$. Clopidogrel was used as a positive control and this compound also reduced inflammatory cell infiltration $(P<0.05)$ and suppressed formation of microthrombi $(P<0.01)$, as compared with the CME model group. Ligustrazine alone exerted a preventative effect against microthrombi $(P<0.01)$, but had no significant effect on myocardial necrosis and inflammatory cell infiltration, as compared with the CME model group $(P>0.05)$. Berberine 

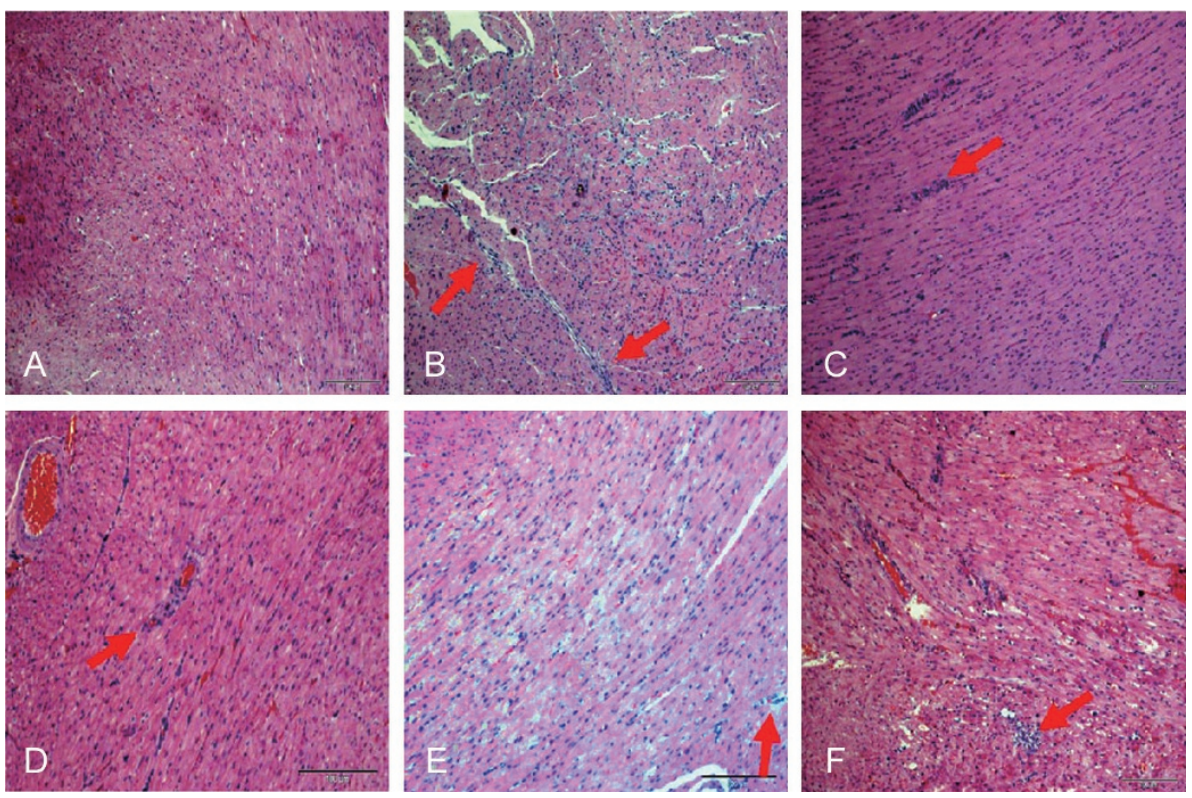

Figure 1. Visualization of inflammatory cellular infiltrates (red arrows) by hematoxylin and eosin staining $(\times 200 ;$ bar=100 $\mu \mathrm{m})$. Rat heart tissue sections are shown from the sham $(A)$, coronary microembolization $(C M E)$ model $(B)$, ligustrazine $(C)$, berberine $(D), L+B(E)$, clopidogrel treatment groups $(F)$. $L$, Ligustrazine; B, Berberine.
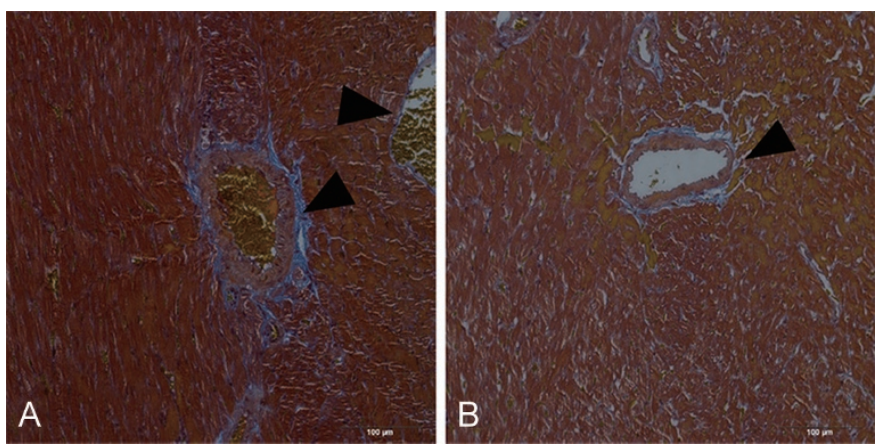

Figure 2. In situ coronary microthrombi were visualized by Carstairs staining. Rat heart tissue sections are shown from the: coronary microembolization (CME) model (A) and sham treatment (B) groups. Multiple arteriolar thrombi (arrow heads) were detected in the CME model group, but not in the sham group. $(\times 200$; bar $=100 \mu \mathrm{m})$.

Table 1. Treatment with ligustrazine and berberine on histology in a rat coronary microembolization model, $24 \mathrm{~h}$ after surgery. Data represent the mean $\pm S E M$ tissue scores in sections that were Heidenhain-stained (for necrosis), hematoxylin and eosin-stained (for infiltration), or Carstairsstained (for microthrombosis). ${ }^{\mathrm{c}} P<0.01$ vs sham. ${ }^{\mathrm{e}} P<0.05,{ }^{\mathrm{f}} P<0.01$ vs the coronary microembolization model. $n=5$.

\begin{tabular}{llcc}
\hline & Necrosis & Infiltration & Microthrombosis (\%) \\
\hline Sham & $0.0 \pm 0.0^{f}$ & $0.0 \pm 0.0^{f}$ & $0.0 \pm 0.0^{f}$ \\
Model & $3.2 \pm 0.2^{c}$ & $2.8 \pm 0.2^{c}$ & $50.0 \pm 3.2^{c}$ \\
Ligustrazine (L) & $2.4 \pm 0.2^{\mathrm{c}}$ & $2.0 \pm 0.3^{\mathrm{c}}$ & $30.8 \pm 3.1^{\text {cf }}$ \\
Berberine (B) & $2.8 \pm 0.4^{\mathrm{c}}$ & $2.2 \pm 0.4^{\mathrm{c}}$ & $40.6 \pm 3.1^{\mathrm{c}}$ \\
L+B & $1.4 \pm 0.2^{\mathrm{cf}}$ & $1.4 \pm 0.2^{\mathrm{cf}}$ & $27.6 \pm 2.3^{\mathrm{cf}}$ \\
Clopidogrel & $2.2 \pm 0.2^{\text {cf }}$ & $1.6 \pm 0.2^{\text {bf }}$ & $29.4 \pm 2.4^{\text {cf }}$ \\
\hline
\end{tabular}

produced no significant reductions of myocardial necrosis, inflammatory cell infiltration, or microthrombi $(P>0.05)$.

\section{Cardiac function}

The effects of treatment with $\mathrm{L}+\mathrm{B}$ on the cardiovascular physiology of rats subjected to CME were examined by measuring various hemodynamic parameters. The results are summarized in Table 2. We found no significant change in HR between the study groups $(P>0.05)$. Compared to the sham group, rats in the CME model group displayed a marked increase in LVEDP $(P<0.01)$ and a decrease in LVSP $(P<0.01)$, $+\mathrm{d} p / \mathrm{d} t_{\max }(P<0.01)$, and $-\mathrm{d} p / \mathrm{d} t_{\max }(P<0.05)$. Pretreatment with L+B significantly decreased LVEDP $(P<0.01)$ and increased LVSP $(P<0.01)$ and $\pm \mathrm{d} p / \mathrm{d} t_{\max }(P<0.05)$, as compared to the CME model group. Similar changes were observed in the clopidogrel group, with no significant difference between the sham and the $\mathrm{L}+\mathrm{B}$ groups $(P>0.05)$. CME rats pretreated with ligustrazine alone exhibited a significant improvement in $+\mathrm{d} p / \mathrm{d} t_{\max }(\mathrm{P}<0.05)$ and a reduction in LVEDP $(P<0.05)$, and the CME rats pretreated with berberine alone had a decreased LVEDP $(P<0.05)$, as compared with untreated CME model rats. These results suggest that pretreatment with $\mathrm{L}+\mathrm{B}$ improves cardiac function in this rat model of CME.

\section{Levels of serum CK-MB}

To further evaluate the cardioprotective effect of $L+B$ in this $\mathrm{CME}$ model, the level of CK-MB in serum was measured after $24 \mathrm{~h}$. This analysis showed that the level of CK-MB was significantly higher in the CME model group than in the sham group $(P<0.01)$. As compared with the CME model group, $\mathrm{L}+\mathrm{B}$ treatment reduced the serum CK-MB level significantly $(P<0.01)$. CME rats pretreated with ligustrazine alone also 

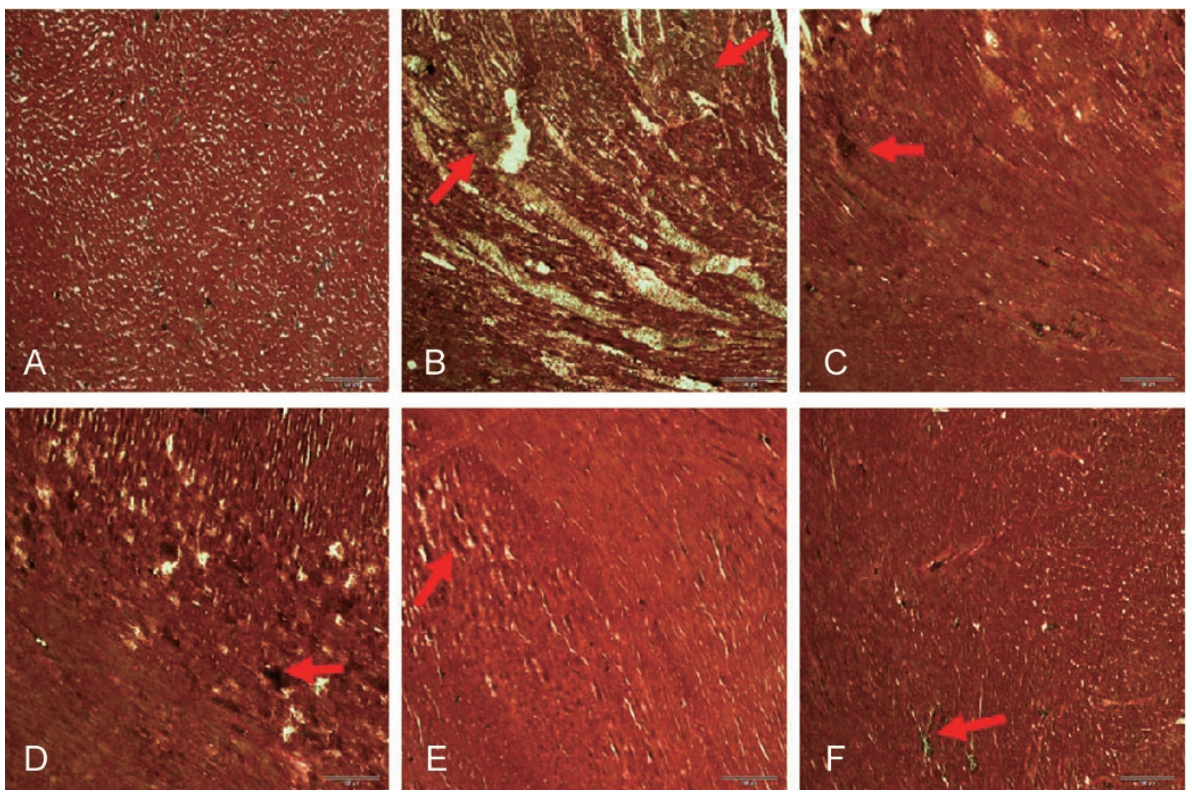

Figure 3. Myocardial necrosis (red arrows) was visualized by Heidenhain staining. Rat heart tissue sections are shown from the the sham (A), coronary microembolization $(C M E)$ model $(B)$, ligustrazine $(C)$, berberine $(D), L+B(E)$, clopidogrel treatment groups $(F)$. L, Ligustrazine; B, Berberine. Necrotic myocardium was stained brown to black. $(\times 200$; bar $=100 \mu \mathrm{m})$.

Table 2. Treatment effects on cardiac function, assessed hemodynamically $24 \mathrm{~h}$ after surgery in a rat coronary microembolization model. Data are expressed as mean \pm SEM. $n=6 .{ }^{b} P<0.05,{ }^{c} P<0.01$ vs sham. ${ }^{e} P<0.05,{ }^{f} P<0.01$ vs the coronary microembolization model.

\begin{tabular}{|c|c|c|c|c|c|}
\hline & LVSP (mmHg) & LVEDP (mmHg) & HR (beat/min) & $+\mathrm{dp} / \mathrm{d} t_{\max }(\mathrm{mmHg} / \mathrm{s})$ & $-\mathrm{dp} / \mathrm{d} t_{\max }(\mathrm{mmHg} / \mathrm{s})$ \\
\hline Sham & $134 \pm 15^{e}$ & $-9.4 \pm 5.7^{f}$ & $508 \pm 17$ & $10188 \pm 994^{f}$ & $-7327 \pm 1380^{e}$ \\
\hline Model & $100 \pm 20^{c}$ & $7.7 \pm 5.4^{c}$ & $495 \pm 24$ & $6510 \pm 1919^{c}$ & $-4639 \pm 1761^{b}$ \\
\hline Berberine (B) & $118 \pm 16$ & $-1.7 \pm 2.6^{e}$ & $504 \pm 16$ & $8602 \pm 779$ & $-6395 \pm 1307$ \\
\hline$L+B$ & $134 \pm 8^{f}$ & $-3.6 \pm 6.6^{f}$ & $509 \pm 12$ & $10110 \pm 445^{e}$ & $-7194 \pm 1076^{e}$ \\
\hline
\end{tabular}

LVSP, left ventricular systolic pressure; LVEDP, left ventricular end-diastolic pressure; HR, heart rate; $+\mathrm{dp} / \mathrm{d} t_{\max }$, the maximum rate of increase of the LVSP; $-\mathrm{dp} / \mathrm{d} t_{\max }$, the maximum rate of decrease of the LVSP.

Table 3. Effects of ligustrazine and berberine on the serum concentration of creatine kinase MB fraction in a rat coronary microembolization model, $24 \mathrm{~h}$ after surgery. Data are expressed as mean \pm SEM. $n=8 .{ }^{\circ} P<0.01 \mathrm{vs}$ sham. ${ }^{e} P<0.05,{ }^{f} P<0.01$ vs the coronary microembolization model.

\begin{tabular}{lc}
\hline Groups & Creatine kinase MB fraction (IU/L) \\
\hline Sham & $674 \pm 114^{f}$ \\
Model & $1545 \pm 229^{\circ}$ \\
Ligustrazine (L) & $1022 \pm 188^{\mathrm{e}}$ \\
Berberine (B) & $1348 \pm 223$ \\
L+B & $867 \pm 223^{f}$ \\
Clopidogrel & $1012 \pm 210^{\mathrm{e}}$ \\
\hline
\end{tabular}

exhibited a significant reduction in CK-MB $(P<0.05)$, while there was no significant change in $\mathrm{CK}-\mathrm{MB}$ in the group treated with berberine only $(P>0.05)$. The results from the clopidogrel group were similar to those of the ligustrazine group.

\section{Plasma levels of vWF and ET-1}

To evaluate the protective function of $\mathrm{L}+\mathrm{B}$ on the endothelium, plasma levels of vWF and ET-1 were measured one day after exposure to sodium laurate. The CME model group exhibited a significant increase in vWF and ET-1 levels, as compared to the sham group $(P<0.05$; Figure 4$)$. This increase in $v W F$ and ET-1 was significantly attenuated in the clopidogrel, L+B, ligustrazine, and berberine treatment groups $(P<0.05)$. vWF and ET-1 levels were similar after clopidogrel and L+B treatments $(P>0.05)$. However, the vWF levels in rats treated with clopidogrel, $\mathrm{L}+\mathrm{B}$, ligustrazine, and berberine were still significantly higher than those observed in the sham group $(P<0.05)$.

\section{Platelet activation}

Plasma P-selectin and cAMP levels, as well as PRI, were examined to investigate the effect of $\mathrm{L}+\mathrm{B}$ on ADP-induced platelet 

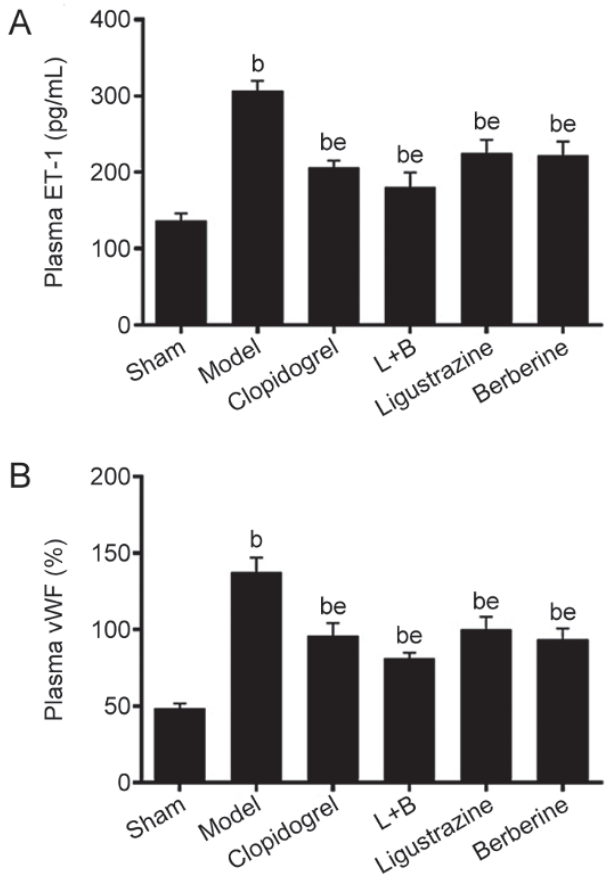

Figure 4. Plasma levels of von Willebrand factor (VWF) and endothelin-1 (ET-1) after ligustrazine and berberine treatment. Data are expressed as mean \pm SEM. $n=6$. ${ }^{\mathrm{b}} P<0.05$ vs sham. ${ }^{\mathrm{e}} P<0.05$ vs coronary microembolization (CME) model.

activation. As shown in Figure 5, PRI was significantly lower in the $\mathrm{L}+\mathrm{B}$, ligustrazine, and clopidogrel groups $(P<0.05)$ than in the CME model group. The plasma P-selectin level was significantly higher in the CME model group, as compared with the sham group $(P<0.05)$. Treatment with $\mathrm{L}+\mathrm{B}$ reduced the plasma P-selectin level significantly $(P<0.05)$, as did clopidogrel. Furthermore, the CME model group had a lower plasma cAMP level than the sham group $(P<0.05)$ and cAMP levels were significantly increased in the $\mathrm{L}+\mathrm{B}$ and clopidogrel groups $(P<0.05)$.

\section{Serum inflammatory mediator levels}

Serum TNFa, IL-1 $\beta$, ICAM-1, and RANTES levels were measured as indices of a systemic inflammatory response. Serum levels of these mediators were increased significantly in the CME model group, as compared with the sham group $(P<0.05$, Figure 6). Treatment with $L+B$ significantly attenuated this increase in TNFa, IL-1 $\beta$, ICAM-1, and RANTES levels $(P<$ 0.05). Clopidogrel decreased TNFa, ICAM-1, and RANTES, but not IL-1 $\beta$, levels, as compared with the CME model group $(P<0.05)$. Ligustrazine alone also decreased serum TNFa, and berberine alone significantly reduced RANTES, as compared with the CME model group ( $P<0.05$ for both comparisons). The level of RANTES in the L+B group was significantly lower than that of the ligustrazine group $(P<0.05)$.

Heart expression of TNF $\alpha$, IL-1 $\beta$, ICAM-1, and RANTES proteins Levels of TNFa, IL-1 $\beta$, ICAM-1, and RANTES proteins in rat hearts were calculated in relation to the constitutively
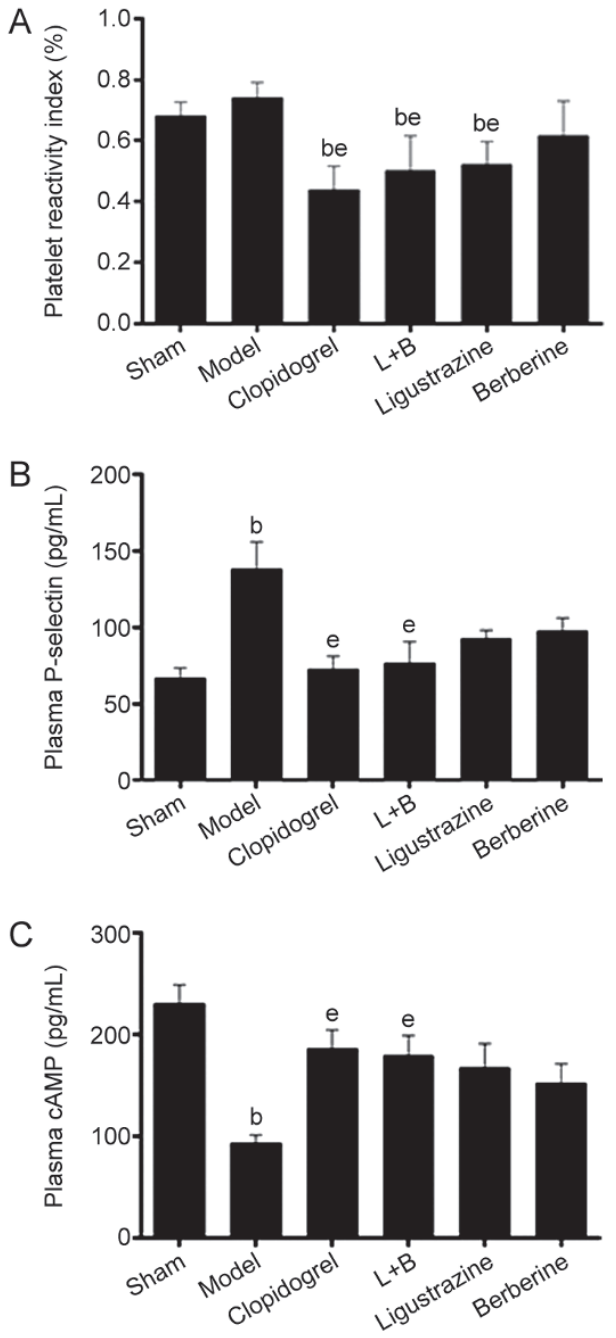

Figure 5. Effect of treatment with ligustrazine and berberine $(L+B)$ on platelet activation. Data are expressed as mean \pm SEM. $n=6$. ${ }^{b} P<0.05$ vs sham. ${ }^{e} P<0.05$ vs coronary microembolization (CME) model.

expressed GAPDH protein as indicators of the local cardiac inflammatory response. As expected, their expression was increased in the CME model group, as compared with the sham group $(P<0.05$; Figure 7). Pretreatment with clopidogrel or $\mathrm{L}+\mathrm{B}$ significantly attenuated the increased expression of all four proteins $(P<0.05)$, although the expression of TNFa, IL-1 $\beta$, and ICAM-1 in the clopidogrel group remained higher than that observed in the sham group $(P<0.05)$. Meanwhile, the expression of TNFa, IL-1 $\beta$, ICAM-1, and RANTES in the $L+B$ group did not differ significantly from that of the sham group $(P>0.05)$. Interestingly, the level of IL- $1 \beta$ in the $L+B$ group was significantly lower than the levels observed in the groups treated with ligustrazine or berberine only $(P<0.05)$.

\section{Discussion}

Over the past three decades, an increasing number of studies have identified disorders in the function and structure of the coronary microcirculation in many clinical conditions. These disorders represent critical contributors to myocardial 

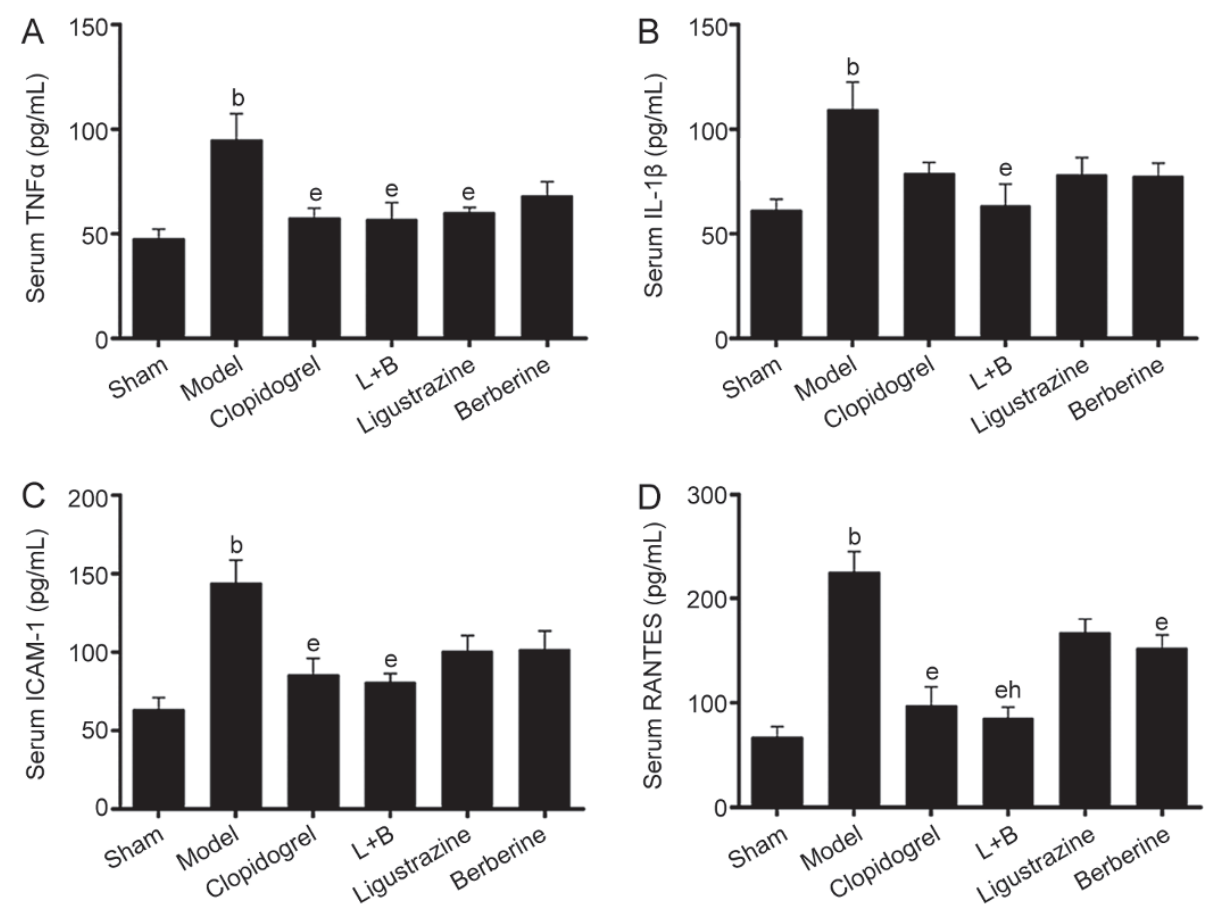

Figure 6. Effect of treatment with ligustrazine and berberine $(L+B)$ on serum inflammatory mediators. Data are expressed as mean $\pm S E M$. $n=6$. ${ }^{\mathrm{b}} \mathrm{P}<0.05$ vs sham. ${ }^{\mathrm{e}} \mathrm{P}<0.05$ vs coronary microembolization $(\mathrm{CME})$ model. ${ }^{\mathrm{h}} \mathrm{P}<0.05$ vs ligustrazine or berberine alone.

A
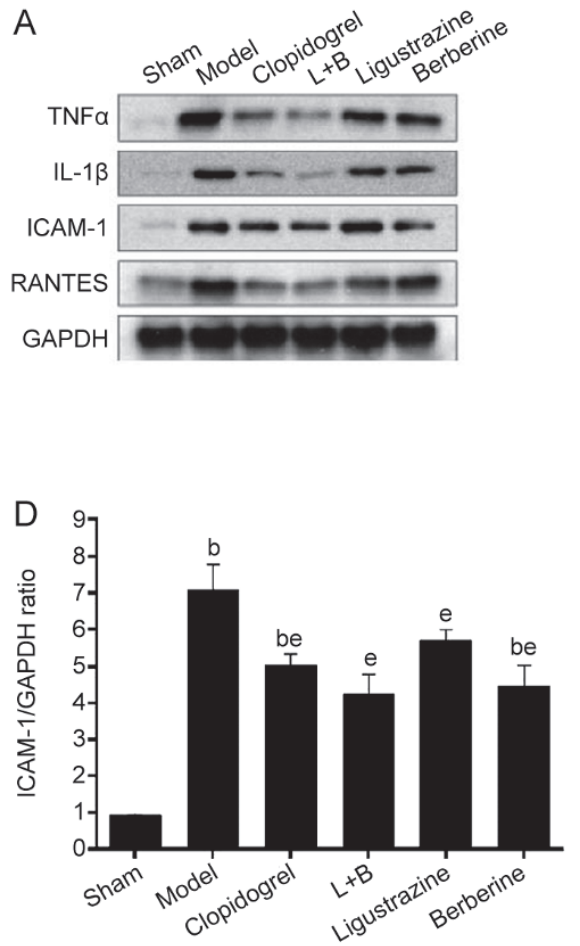
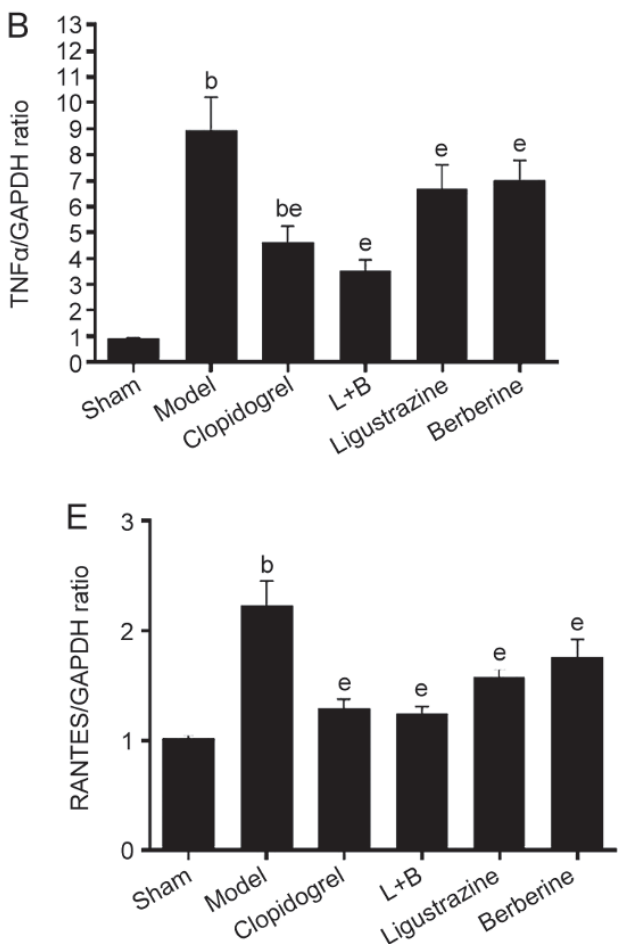

Figure 7. Western blot images of tumor necrosis factor alpha (TNF- $\alpha$ ), interleukin (IL)-1 $\beta$, intracellular adhesion molecule-1 (ICAM-1), and regulated on activation, normal T cell-expressed and secreted (RANTES) protein levels in myocardial cells. Densitometric data are expressed as mean \pm SEM. $n=6$. ${ }^{\mathrm{b}} \mathrm{P}<0.05$ vs sham. ${ }^{\mathrm{e}} \mathrm{P}<0.05$ vs coronary microembolization $(\mathrm{CME})$ model. ${ }^{\mathrm{h}} \mathrm{P}<0.05$ vs ligustrazine or berberine alone.

ischemia and have become important therapeutic targets ${ }^{[21]}$. Therefore, the establishment of an animal model of CME that mimics the clinical pathology is important for studies of conditions where coronary microvascular dysfunction occurs, such 
as microvascular angina.

In the present study, a CME model was successfully established in rats by the LV injection of sodium laurate, as evidenced by endothelial injury, in situ coronary thrombi, myocardial necrosis with obvious inflammatory cell infiltration, and LV dysfunction. Compared with previous CME models induced by the LV injection of homologous microthrombotic particles ${ }^{[16-17]}$, the endothelial injury in the present model was induced first by sodium laurate, triggering platelet adhesion and aggregation that resulted in in situ thrombosis within coronary arterioles. In addition, endothelial injury or thrombi formation were not observed in the large coronary arteries. These animal pathologies were therefore more similar to those observed in patients, as compared with the previously reported models. Our findings also indicated that sodium laurate had dose-dependent effects on the endothelium. In a preliminary experiment, $0.2 \mathrm{~mL}$ of $1 \mathrm{~g} / \mathrm{L}$ sodium laurate was injected into the LV and no thrombi were observed in the coronary arterioles (diameter $\leq 100 \mu \mathrm{m}$ ). However, the dose of 0.2 $\mathrm{mL}$ of $2 \mathrm{~g} / \mathrm{L}$ sodium laurate used in the present study caused endothelial injury and thrombotic occlusion of the coronary arterioles.

Clopidogrel inhibits platelet activation by blocking the $\mathrm{P}_{2} \mathrm{Y}_{12}$ platelet receptor and it is one of the most widely used antiplatelet drugs for patients with coronary artery disease. The present study showed that, in addition to its antiplatelet effects, clopidogrel showed anti-inflammatory activity; this was evidenced by the decreased serum levels of TNFa, ICAM-1, and RANTES, and heart tissue expression of TNFa, ICAM-1, IL-1 $\beta$, and RANTES proteins. These findings were consistent with previous studies ${ }^{[22-25]}$ and indicated that the cardioprotective effects of clopidogrel observed in the present study may be, in part, attributed to these anti-inflammatory effects.

Both ligustrazine and berberine are the component of Chinese herbs that have been used for more than two decades for the treatment of atherosclerotic and inflammatory diseases $^{[26-29]}$. Our study demonstrated that ligustrazine alone improved cardiac function in CME rats, as evidenced by an increased $+\mathrm{d} p / \mathrm{d} t_{\max }$, decreased LVEDP, and decreased serum CK-MB level. Berberine also decreased the LVEDP, but had no obvious impact on CK-MB levels. Both the histopathological findings and hemodynamic results indicated that use of ligustrazine and berberine in combination produced greater effects than either agent alone. Our study identified a unique antiplatelet effect of ligustrazine (reduced PRI), while the marked anti-inflammatory effect of berberine may contribute to its cardioprotective activity. Interestingly, the use of ligustrazine and berberine in combination enhanced these antiplatelet and anti-inflammatory effects. A series of previous studies by Shang et $a l^{[30-31]}$ showed that Chinese herbs that activated blood circulation produced synergistic effects when administered with detoxifying herbs. The findings of the present study indicated that ligustrazine and berberine may also have synergistic effects, although the mechanism involved remains to be elucidated.
P2 $\mathrm{Y}_{12}$ inhibitors such as clopidogrel are associated with increased survival rates in patients with coronary microvascular dysfunction ${ }^{[32-33]}$. The present study showed that treatment with the combination of ligustrazine and berberine produced a similar effect as clopidogrel, blocking the $\mathrm{P}_{2} \mathrm{Y}_{12}$ platelet receptor and increasing CAMP and VASP-239 phosphorylation. Although ligustrazine alone also inhibited the $\mathrm{P}_{2} \mathrm{Y}_{12}$ platelet receptor, this effect was enhanced when it was administered with berberine.

Previous studies ${ }^{[34-35]}$ have demonstrated the pivotal role of inflammation in adverse LV function. Patients with heart failure often have elevated levels of pro-inflammatory cytokines and chemokines. Among the cytokines, TNFa has been implicated in the development of LV dysfunction ${ }^{[36]}$. IL-1 $\beta$ increases dynamically following CME and is associated with impaired cardiac function ${ }^{[37]}$. RANTES has been identified as a specific marker for unstable angina pectoris by several studies ${ }^{[38-39]}$. The adhesion molecule, ICAM-1, also represents a marker of slow coronary flow caused by microvascular dysfunction ${ }^{[40-41]}$. Therefore, we chose these four molecular factors for the present evaluation of CME-related inflammatory responses. Although only some of these markers were reduced in rats treated with ligustrazine or berberine separately, all four were significantly affected in animals treated with these compounds in combination. These effects were greater than those of clopidogrel, suggesting that the inflammatory response reflected not only platelet activation, but also vascular endothelial injury. Plasma levels of both ET-1 and vWF, which are markers of vascular endothelial injury in the cardiovascular system, were reduced in rats treated with the combination of ligustrazine and berberine.

Coronary microvascular dysfunction can present clinically as chest pain in individuals with normal coronary arteriograms (ie, microvascular angina) or in the context of other cardiac disease processes. The assessment and visualization of human coronary microcirculation anatomy is clearly challenging and this creates difficulties in relation to diagnosis and treatment. Therefore, the present study is of great significance for clinical practice and complements the increasing body of clinical research demonstrating the cardioprotective effects of ligustrazine and berberine ${ }^{[42-43]}$.

The present study had some limitations. First, we only investigated the short-term protective effects of the test compounds. Thus, future studies should investigate whether this protective effect is sustained over the longer term. Second, the present study did not explore whether the mechanisms underlying the action of the combination of ligustrazine and berberine were associated with other factors.

In conclusion, our study found that a rat CME model showed cardiac dysfunction, which was alleviated by combined treatment with ligustrazine and berberine. The mechanism underlying this protective effect appeared to involve inhibition of endothelial injury, reduced ADP-mediated platelet activation, and anti-inflammatory effects. The combined use of ligustrazine and berberine might provide a reasonable phytomedicine for the treatment of patients with diseases 
involving coronary microvascular dysfunction.

\section{Acknowledgements}

This work was supported by the National Natural Science Foundation of China (№ 81030063 to Da-zhuo SHI).

\section{Author contribution}

Da-zhuo SHI and Xiao-juan MA designed the study and revised the manuscript; Ying ZHANG wrote the manuscript and performed the animal experiments; Chun-yu GUO, Mingming WANG, Na KOU, Hua QU, and Hui-min MAO contributed to the molecular biology experiments.

\section{References}

1 Erbel R, Heusch G. Coronary microembolization. J Am Coll Cardiol 2000; 36: 22-4.

2 Camici PG, Crea F. Coronary microvascular dysfunction. N Engl J Med 2007; 356: 830-40.

3 Gulati M, Cooper-DeHoff RM, McClure C, Johnson BD, Shaw LJ, Handberg EM, et al. Adverse cardiovascular outcomes in women with nonobstructive coronary artery disease: a report from the Women's Ischemia Syndrome Evaluation Study and the St James Women Take Heart Project. Arch Intern Med 2009; 169: 843-50.

4 Davì G, Patrono C. Platelet activation and atherothrombosis. New Engl J Med 2007; 357: 2482-94.

5 Wang $\mathrm{H}$, Zhong WJ, Huang MW, Wu XY Chen H. Efficacy of dual antiplatelet therapy combined with Naoxintong capsules following coronary microembolization induced by homologous microthrombi in rats. Chin J Integr Med 2011; 17: 917-24.

6 Liu SY, Sylvester DM. Antiplatelet activity of tetramethylpyrazine. Thromb Res 1994; 75: 51-62.

7 Sheu JR, Kan YC, Hung WC, Ko WC, Yen MH. Mechanisms involved in the antiplatelet activity of tetramethylpyrazine in human platelets. Thromb Res 1997; 88: 259-70.

8 Li Y, Song P, Zhu Q, Yin QY, Ji JW, Li W, et al. Liguzinediol improved the heart function and inhibited myocardial cell apoptosis in rats with heart failure. Acta Pharmacol Sin 2014; 35: 1257-64.

9 Ji XX, Song XL, Qian W, Yu XL, Zhu JY. Effects and mechanism of action of ligustrazine on isoprenaline-induced cardiomyocyte hypertrophy. Cell Biochem Biophys 2014; 70: 1513-8.

10 Kong WJ, Wei J, Abidi P, Lin MH, Inaba S, Li C, et al. Berberine is a novel cholesterol-lowering drug working through a unique mechanism distinct from statins. Nat Med 2004; 10: 1344-51.

11 Huang ZQ, Han ZH, Ye BZ, Dai ZY, Shan PR, Lu ZQ, et al. Berberine alleviates cardiac ischemia/reperfusion injury by inhibiting excessive autophagy in cardiomyocytes. Eur J Pharmacol 2015; 762: 1-10.

12 Yu LM, Li FJ, Zhao GL, Yang Y, Jin ZX, Zhai MG, et al. Protective effect of berberine against myocardial ischemia reperfusion injury: role of Notch1/Hes1-PTEN/Akt signaling. Apoptosis 2015; 20: 796-810.

13 Chen KK, Li GH, Geng FH, Zhang Z, Li JN, Yang M, et al. Berberine reduces ischemia/reperfusion-induced myocardial apoptosis via activating AMPK and PI3K-Akt signaling in diabetic rats. Apoptosis 2014; 19: 946-57.

14 Zhang YJ, Yang SH, Li MH, Iqbal J, Bouranta CV, Mi QY, et al. Berberine attenuates adverse left ventricular remodeling and cardiac dysfunction after acute myocardial infarction in rats: role of autophagy. Clin Exp Pharmacol Physiol 2014; 41: 995-1002.

15 Ma XJ, Guo CY, Yin HJ, Liu Y, Shi DZ. Effect of activating blood circulation or activating blood circulation and detoxification on platelet activation, inflammation, and coagulation status in acute myocardial infarction rats. Chin J Integr Tradit West Med 2014; 34: 1329-34.

16 Li SM, Zhong SS, Zeng K, Luo YK, Zhang FL, Sun XD, et al. Blockade of NF-KB by pyrrolidine dithiocarbamate attenuates myocardial inflammatory response and ventricular dysfunction following coronary microembolization induced by homologous microthrombi in rats. Basic Res Cardiol 2010; 105: 139-50.

17 Bai YP, Hu LQ, Wu J, Gu Y, Li L, Gao B, et al. Effects of intravenous diltiazem in a rat model of experimental coronary thrombotic microembolism. Exp Ther Med 2013; 6: 873-82.

18 Shen CX, Liang C, Chen LL, Qian JY, Wang KQ, Chen HZ, et al. The establishment of a new model of rat coronary microthrombosis by coronary sodium laurate injection. Chin J Arterioscl 2005; 13: $447-$ 50.

19 Toshima Y, Satoh S, Ikegaki I, Asano T. A new model of cerebral microthrombosis in rats and the neuroprotective effect of a Rhokinase inhibitor. Stroke 2000; 31: 2245-50.

20 Schumacher WA, Bostwick JS, Ogletree ML, Stewart AB, Steinbacher $\mathrm{TE}$, Hua J, et al. Biomarker optimization to track the antithrombotic and hemostatic effects of clopidogrel in rats. J Pharmacol Exp Ther 2007; 322: 369-77.

21 Duran JR 3rd, Taffet G. Coronary microvascular dysfunction. N Engl J Med 2007; 356: 2324-5.

22 Molero L, López-Farré A, Mateos-Cáceres PJ, Fernández-Sánchez R, Maestro ML, Silva J, et al. Effect of clopidogrel on the expression of inflammatory markers in rabbit ischemic coronary artery. $\mathrm{Br} J$ Pharmacol 2005; 146: 419-24.

23 Wong KSL, Chen C, Fu J, Chang HM, Suwanwela NC, Huang YN, et al. Clopidogrel plus aspirin versus aspirin alone for reducing embolisation in patients with acute symptomatic cerebral or carotid artery stenosis (CLAIR study): a randomised, open-label, blinded-endpoint trial. Lancet Neurol 2010; 9: 489-97.

24 Cardoso RN, Benjo AM, DiNicolantonio JJ, Garcia DC, Macedo FY, El-Hayek G, et al. Incidence of cardiovascular events and gastrointestinal bleeding in patients receiving clopidogrel with and without proton pump inhibitors: an updated meta-analysis. Open Heart 2015; 2: e000248.

25 Choi H, Ryu J, Seo H, Kang M, Kim E. Is a high maintenance dose of clopidogrel suitable for overcoming clopidogrel resistance in patients?. Int J Clin Pharm 2015; 37: 758-61.

26 Chen YJ, Huang CS, Wang F, Gong JY, Pan ZH. Effect of ligustrazine hydrochloride on coagulation reaction and inflammation reaction in single valve replacement patients with rheumatic heart disease undergoing cardiopulmonary bypass. Chin J Integr Tradit West Med 2014; 34: 531-5.

27 Ni XJ, Liu SN, Guo XF. Medium- and long-term efficacy of ligustrazine plus conventional medication on ischemic stroke: a systematic review and meta-analysis. J Tradit Chin Med 2013; 33: 715-20.

28 Yan HM, Xia MF, Wang Y, Chang XX, Yao XZ, Rao SX, et al. Efficacy of berberine in patients with non-alcoholic fatty liver disease. PLoS One 2015; 10: e0134172.

29 Zhang JF, Cao HL, Zhang B, Cao HW, Xu XQ, Ruan H, et al. Berberine potently attenuates intestinal polyps growth in ApcMin mice and familial adenomatous polyposis patients through inhibition of Wnt signaling. J Cell Mol Med 2013; 17: 1484-93.

30 Shang XB, Wu XZ, Li DH, Guo SD. Experimental study on synergistic action of Huoxue Huayu herbs on Qingjie Tongxia Herbs I. Chin J Surg Integr Tradit West Med 2006; 12: 91-4.

31 Shang XB, Wu XZ, Xue XP, Yang XZ, Li DH, Guo SD. Experimental studies on synergistic effect of Huoxue Huayu herbs on Qingjie Tongxia Herbs IV. Chin J Surg Integr Tradit West Med 2006; 12: 254-7.

32 Park SD, Baek YS, Woo SI, Kim SH, Shin SH, Kim DH, et al. Comparing 
the effect of clopidogrel versus ticagrelor on coronary microvascular dysfunction in acute coronary syndrome patients (TIME trial): study protocol for a randomized controlled trial. Trials 2014; 15: 151.

33 Willoughby SR, Luu LJ, Cameron JD, Nelson AJ, Schultz CD, Worthley $\mathrm{SG}$, et al. Clopidogrel improves microvascular endothelial function in subjects with stable coronary artery disease. Heart Lung Circ 2014; 23: 534-41.

34 Sola S, Mir MQ, Lerakis S, Tandon N, Khan BV. Atorvastatin improves left ventricular systolic function and serum markers of inflammation in nonischemic heart failure. J Am Coll Cardiol 2006; 47: 332-7.

35 Dörge H, Schulz R, Belosjorow S, Post H, van de Sand A, Konietzka I, et al. Coronary microembolization: the role of TNF- $\alpha$ in contractile dysfunction. J Mol Cell Cardiol 2002; 34: 51-62.

36 Yamada T, Matsumori A, Sasayama S. Therapeutic effect of antitumor necrosis factor-alpha antibody on the murine model of viral myocarditis induced by encephalomyocarditis virus. Circulation 1994; 89: 846-51.

37 Li L, Zhao XM, Lu YG, Huang WQ, Wen WM. Altered expression of proand anti-inflammatory cytokines is associated with reduced cardiac function in rats following coronary microembolization. Mol Cell
Biochem 2010; 342: 183-90.

38 Kraaijeveld AO, de Jager SCA, De Jager WJ, Prakken BJ, McColl SR, Haspels I, et al. CC chemokine ligand-5 (CCL5/RANTES) and CC chemokine ligand-18 (CCL18/PARC) are specific markers of refractory unstable angina pectoris and are transiently raised during severe ischemic symptoms. Circulation 2007; 116: 1931-41.

39 Cipriani S, Francisci D, Mencarelli A, Renga B, Schiaroli E, D’Amore $C$, et al. Efficacy of the CCR5 antagonist maraviroc in reducing early, ritonavir-induced atherogenesis and advanced plaque progression in mice. Circulation 2013; 127: 2114-24.

40 Wagner DD. New links between inflammation and thrombosis. Arterioscler Thromb Vasc Biol 2005; 25: 1321-4.

41 Wagner DD, Burger PC. Platelets in inflammation and thrombosis. Arterioscler Thromb Vasc Biol 2003; 23: 2131-7.

42 Liu GX. Meta-analysis of ligustrazine injection in the treatment of unstable angina pectoris. Modern J Integr Tradit Chin West Med 2012; 17: 4-6.

43 Dong H, Yan Z, Li Z, Lu FE. The effects of berberine on blood lipids: a systemic review and meta-analysis of randomized controlled trials. Planta Med 2013; 79: 437-46. 